\title{
BML-275, an AMPK inhibitor, induces DNA damage, G2/M arrest and apoptosis in human pancreatic cancer cells
}

\author{
HONG-QUAN DUONG ${ }^{1,3}$, JAE SEOK HWANG ${ }^{1,4}$, HEE JEONG KIM ${ }^{1}$, YEON-SUN SEONG ${ }^{3}$ and INSOO BAE ${ }^{1-3}$ \\ Departments of ${ }^{1}$ Oncology and ${ }^{2}$ Radiation Medicine, Lombardi Comprehensive Cancer Center, Georgetown University, \\ Washington, DC, USA; ${ }^{3}$ WCU (World Class University) Research Center of Nanobiomedical Science, Dankook University, \\ Cheonan; ${ }^{4}$ Department of Internal Medicine, Keimyung University College of Medicine, Daegu, Republic of Korea
}

Received July 18, 2012; Accepted September 7, 2012

DOI: 10.3892/ijo.2012.1672

\begin{abstract}
Adenosine monophosphate-activated protein kinase (AMPK) is a principal intracellular energy sensor which regulates energy producing pathways and energy requiring pathways when the cellular AMP/ATP ratio is altered. BML-275 (compound C), a well-known inhibitor of AMPK, has been found to induce apoptosis in myeloma, glioma and prostate cancer cells. However, the mechanisms responsible for the selective apoptotic effect(s) by BML-275 in cancer cells remain unknown. In the present study, BML-275 was investigated for its antitumor effect(s) in human pancreatic cancer cell lines. BML-275 inhibited the cell proliferation of 4 human pancreatic cancer cell lines (MIA PaCa-2, Panc-1, Colo-357 and AsPC-1). In addition, BML-275 significantly increased the generation of intracellular reactive oxygen species (ROS), followed by induction of DNA damage signaling and apoptosis. Furthermore, BML-275 induced cell cycle arrest in the G2/M phase. The inhibition of ROS generation by $\mathrm{N}$-acetyl cysteine (NAC) significantly prevented the induction of DNA damage and apoptosis, but failed to prevent the induction of $\mathrm{G} 2 / \mathrm{M}$ arrest by BML-275. Small interfering RNA (siRNA)-mediated knockdown of AMPK $\alpha$ increased the generation of intracellular ROS, DNA damage signaling and apoptosis without cell cycle arrest at the G2/M phase. These findings suggest that BML-275 exerts its antitumor effects by inducing ROS generation, DNA damage and apoptosis via inhibition of the AMPK pathway and by inducing G2/M arrest via a pathway independent of AMPK, implicating its potential application as an antitumor agent for pancreatic cancer.
\end{abstract}

Correspondence to: Dr Insoo Bae, Department of Oncology and Radiation Medicine, Lombardi Comprehensive Cancer Center, Georgetown University, Washington, DC 20057, USA

E-mail: ib42@georgetown.edu

Dr Yeon-Sun Seong, WCU Research Center of Nanobiomedical Science, Dankook University, Cheonan 330-714, Republic of Korea E-mail: seongys@chol.com

Key words: adenosine monophosphate-activated protein kinase, BML-275, DNA damage, G2/M arrest, apoptosis, pancreatic cancer

\section{Introduction}

Pancreatic adenocarcinoma is one of the most lethal and poorly understood human malignancies. Because of the lack of effective systemic therapies the 5-year survival rate for patients with pancreatic adenocarcinoma has remained at 1-3\% without a change over the past 25 years $(1,2)$. To date, the only potential curative means is surgical resection, of which only $20 \%$ of patients are eligible. Alternative therapies, such as radiotherapy and chemotherapy remain largely ineffective. Therefore, the development and evaluation of novel targeted therapeutic agents that reduce the intrinsic drug resistance of this disease poses one of the greatest challenges in pancreatic cancer research and other intractable cancers.

AMP-activated protein kinase (AMPK), a serine/threonine kinase, is a highly conserved sensor of cellular energy status in eukaryotes and is widely known as a regulator of cell metabolism (3). AMPK is a heterotrimeric protein consisting of a catalytic $\alpha$-subunit and regulatory $\beta$ - $/ \gamma$-subunits $(4,5)$. It is phosphorylated at Thr172 in response to an increase in the ratio of AMP-to-ATP within its activation domain of $\alpha$-subunit by upstream kinases LKB1 (6-8) and calmodulin-dependent protein kinase kinase $\beta$ (CaMKK $\beta$ ) (9-11). Several previous studies show that excessive AMPK activation by treatment of AMPK activator (such as Metformin, 5-aminoimidazole4-carboxamide riboside (AICAR) or A769662) inhibits the growth and/or survival of various cancer cell lines (12-19). Moreover, BML-275 (compound C), a potent, selective, and reversible ATP-competitive inhibitor of AMPK induces cell death in various types of cancers including myeloma, glioma, prostate and breast carcinoma cells (20-23). In addition, inhibition of AMPK pathway by compound $\mathrm{C}$ sensitizes apoptosis by co-treatment with tumor necrosis factor-related apoptosis-inducing ligand (TRAIL), doxorubicin or cisplatin in human renal, leukemia, gastric carcinoma, colon carcinoma, and cervix adenocarcinoma cell lines (24-26). Therefore, pharmacological inhibition of AMPK activity might be potentially useful in therapy of human solid tumors. However, the effect of AMPK inhibition of pancreatic cancer cell proliferation or survival has not been investigated.

Cell cycle deregulation resulting in uncontrolled cell proliferation is the one of the most frequent alterations that occurs during tumor development (27) and targeting of cell cycle 
progression and/or machinery is effective strategy to control aberrant proliferation of cancer cell $(28,29)$. There are two major checkpoints, G1/S and G2/M checkpoints, are known to regulate the cell cycle. The $\mathrm{G} 2 / \mathrm{M}$ checkpoint plays a key role in the maintenance of chromosomal integrity by allowing cells to repair DNA damage before entering mitosis. A key regulator of the cell cycle at $\mathrm{G} 2 / \mathrm{M}$ checkpoint is cyclin dependent kinase 1 (CDK1), especially cell division cycle 2 (Cdc2). Cdc2 activation depends on the dephosphorylation of Tyr15 by Cdc25C (30). In addition, Cdc 2 can be further regulated by GADD45 and 14-3-3 by p53 pathway (31). Reactive oxygen species (ROS) generation causes oxidative stress and has been shown to significantly function to controlling cancer cell survival (32). Oxidation of DNA bases and breakage of DNA strand may occurs as results of oxidative DNA damage and parts of these lesions are converted to DNA double-strand breaks (33-35). BML-275 was reported to induce cell cycle arrest at G2/M-phase and ROS generation in U251 glioma cells (22). Therefore, understanding the molecular mechanisms of BML-275 to sensitize these cells to undergo BML-275-mediated G2/M arrest and apoptosis is an important issue for effective cancer therapy.

In this study, we performed experiments to determine antitumor effect(s) by BML-275 in human pancreatic cancer cell lines. Our results suggest that BML-275 regulates cell survival via targeting AMPK and generating ROS in multiple human pancreatic cancer cells.

\section{Materials and methods}

Cell culture and reagents. MIA PaCa-2, Panc-1, CFPAC-1 and BxPC-3 cells were purchased from American Type Culture Collection (ATCC, Manassas, VA, USA) and AsPC-1, Capan-1 and Colo-357 cells were obtained from Tissue Culture Shared Resource of Georgetown University Lombardi Comprehensive Cancer Center (Washington, DC, USA). Immortal human pancreatic ductal epithelial cells, HPDE6-C7 were acquired from Dr M.S. Tsao (36). AsPC-1, BxPC-3, Capan-1 and Colo357 cells were cultured in RPMI-1640 media supplemented with fetal bovine serum (FBS; 20\% for AsPC-1, 10\% for Colo357, Capan-1 and BxPC-3 cells), $100 \mathrm{U} / \mathrm{ml}$ penicillin, $100 \mu \mathrm{g} /$ $\mathrm{ml}$ streptomycin and $1 \%$ sodium pyruvate. MIA PaCa-2 cells were cultured in Dulbecco's modified Eagle's medium (DMEM) containing $10 \%$ FBS, $2.5 \%$ horse serum (HS), $100 \mathrm{U} / \mathrm{ml}$ penicillin and $100 \mu \mathrm{g} / \mathrm{ml}$ streptomycin. Panc-1 and CFPAC-1 cells were cultured in DMEM containing 10\% FBS, $10 \mathrm{U} / \mathrm{ml}$ penicillin and $10 \mu \mathrm{g} / \mathrm{ml}$ streptomycin. HPDE6-C7 cells were cultured in keratinocyte serum-free (KSF) medium supplemented by an epidermal growth factor and bovine pituitary extract and $1 \mathrm{X}$ antibiotic-antimycotic. Cell culture reagents were purchased from BioWhittaker (Walkersville, MD, USA) and Invitrogen (Carlsbad, CA, USA). BML-275 was purchased from Tocris Bioscience (Ellisville, MO, USA), and A769662 was obtained from LC Laboratories (Woburn, MA, USA).

3-(4,5-dimethylthiazol-2-yl)-2,5-diphenyltetrazolium bromide (MTT) assay. A total of 2,000 human pancreatic cancer cells, counted by the Luna Cell Counter (Logos Biosystems, Gyeonggi-Do, Korea) were plated in 96-well flat-bottom plates and then exposed to test the effects of BML-275 in various concentrations. At the indicated times, $10 \mu \mathrm{l}$ of $1 \mathrm{mg} / \mathrm{ml}$
MTT (Sigma, St. Louis, MO, USA) in PBS was added to each well for $4 \mathrm{~h}$. After centrifugation and removal of the medium, $150 \mu \mathrm{l}$ of DMSO (Sigma) was added to each well to dissolve the formazan crystals. The absorbance was measured at $560 \mathrm{~nm}$ using an ELx808 Absorbance Microplate Reader (BioTek Instruments Inc., Winooski, VT, USA). Absorbance of untreated cells was designated as $100 \%$ and cell survival was expressed as a percentage of this value. Triplicate wells were assayed for each condition and standard deviation (SD) was determined.

Western blot (WB) analysis. Cells were grown to $~ 70 \%$ confluence and reagents were added at the indicated concentrations. After exposure to BML-275 alone or in combination with NAC, cells were lysed in cell lysis buffer containing $20 \mathrm{mM}$ Tris- $\mathrm{HCl}$, $0.5 \mathrm{M} \mathrm{NaCl}, 0.25 \%$ Triton X-100, $1 \mathrm{mM}$ EDTA, $1 \mathrm{mM}$ EGTA, $10 \mathrm{mM} \beta$-glycophosphate, $10 \mathrm{mM} \mathrm{NaF}, 300 \mu \mathrm{M} \mathrm{Na}_{3} \mathrm{VO}_{4}, 1 \mathrm{mM}$ benzamidine, $2 \mu \mathrm{MPMSF}$ and $1 \mathrm{mMDTT}$.Protein concentrations were determined by a BCA protein assay kit (Thermo Scientific, Rockford, IL, USA). Proteins were separated by SDS-PAGE, transferred on to PVDF membranes, blocked in 1X blocking buffer (Sigma) and probed with the following primary antibodies: phospho-ACC (S79), ACC, phospho-AMPK $\alpha$ (T172), AMPK, phospho-ATM (S1981), phospho-CHK2 (T68), phospho-Histone H2A.X (S139), XIAP and Survivin (Cell Signaling Technology, Boston, MA), Bcl2 and Poly-ADP-Ribose-Polymerase (PARP; BD Biosciences, Franklin, NJ, USA) and $\alpha$-tubulin (Sigma). Then, the membranes were incubated with horseradish peroxidase (HRP)-conjugated secondary antibodies (Sigma) and visualized with a chemiluminescence kit (Santa Cruz Biotechnology, Santa Cruz, CA, USA) according to the manufacturer's recommended protocol and exposed with X-ray film (American X-ray and Medical Supply, Jackson, CA, USA).

Clonogenic assay. Human pancreatic cancer cells (4x10 5 cells) were seeded in 60-mm dishes. Twenty-four hours after plating, varying concentrations of the drugs, either as a single agent or in combination, were added to the dishes. After treatment, cells (2,000 cells) were re-seeded in 60-mm dishes (triplicate). Each culture dish was incubated for 14 days and photographed after staining with $0.5 \%$ crystal violet in $1 \mathrm{X}$ PBS including $25 \%$ methanol. Colonies were examined under a light microscope and counted after capturing images by scanner. Colony numbers were calculated according to the percentage of the untreated cells (37).

Flow cytometry. Human pancreatic cancer cell lines were collected after treatment of BML-275 by trypsinization, washed with PBS and fixed overnight in $70 \%$ ethanol at $-20^{\circ} \mathrm{C}$. Cells were incubated with $20 \mu \mathrm{g} / \mathrm{ml}$ propidium iodide and $40 \mu \mathrm{g} / \mathrm{ml}$ RNase A in 1X PBS. Cells were analyzed on a FACSCalibur flow cytometer (Becton Dickinson, San Jose, CA, USA) at the Flow Cytometry and Cell Sorting Shared Resource, Georgetown University Lombardi Comprehensive Cancer Center. The acquired data were analyzed by Cell Quest Pro Analysis software (Becton Dickinson).

Small interfering RNA (siRNA). For the RNA interfering experiment, AMPK $\alpha$-siRNA, 5'-CUGAGUUGCAUAUACUGUA-3' and control-siRNA, 5'-GACGAGCGGCACGUGCACA-3' were 
purchased from Bioneer (Daejeon, Korea). AMPK $\alpha$-siRNA or control-siRNA were transfected into MIA PaCa-2 and Panc-1 cells using Lipofectamine 2000 (Invitrogen) according to the manufacturer's procedure. After $48 \mathrm{~h}$ transfected cells were processed for cell cycle analysis, WB analysis and measurement of ROS generation.

ROS generation. For measurement of ROS generation, human pancreatic cancer cell lines were treated with BML-275 with or without $\mathrm{N}$-acetyl cysteine (NAC) for the indicated times and then loaded with $50 \mu \mathrm{M}$ 2', 7'-dichlorofluorescin diacetate (DCFDA; Molecular Probes, Eugene, OR, USA) and $0.5 \mu \mathrm{g} / \mathrm{ml}$ Hoechst 33342 (HO; Sigma) for $30 \mathrm{~min}$. After rinsing, fluorescent images were taken with fluorescence intensities were obtained with a Fluorocount at excitation/emission wavelengths of $490 / 530 \mathrm{~nm}$ (DCFDA) and 340/425 (HO), and values of ROS generation were obtained by determining the ratio of DCFDA/HO signals per well.

Statistical methods. Statistical comparisons were made using the two-tailed Student's t-test where appropriate. Results were considered significant in at means ${ }^{*} \mathrm{P}<0.05,{ }^{* *} \mathrm{P}<0.01$ and ${ }^{* * *} \mathrm{P}<0.005$. Data were expressed as the mean $\pm \mathrm{SD}$.

\section{Results}

Human pancreatic cancer cells and immortal human pancreatic duct epithelial cells express AMPK $\alpha$. We first examined the total and phosphorylated form of AMPK $\alpha$ in AsPC-1, Panc-1, MIA PaCa-2, Capan-1, CFPAC-1, Colo-357, BxPC-3 and HPDE6-C7 cells. The WB result reveals that all pancreatic cell lines used for this study expressed the levels of both phosphorylatedAMPK and total-AMPK (Fig. 1). Next, we investigated the expression level of AMPK target protein, Acetyl-CoA Carboxylase (ACC). We found that there is relatively good correlation between the levels of phosphorylated-AMPK and phosphorylated-ACC among pancreatic cell lines (Fig. 1). To study the antitumor effect(s) by BML-275, AMPK inhibitor, in pancreatic cancer cells, we chose four pancreatic cancer cell lines: MIA PaCa-2, Panc-1, Colo-357 and AsPC-1 for further studies.

BML-275 induces apoptotic cell death. BML-275 is a potent ATP-mimetic competitive inhibitor of AMPK. In order to explore the antitumor effect(s) by BML-275, MIA PaCa-2, Panc-1, Colon-357 and AsPC-1 cells were treated with different concentrations of BML-275 $(0,1,3,5 \mathrm{and} /$ or $10 \mu \mathrm{M})$ for $48 \mathrm{~h}$ and cell viability were measured by MTT assay. BML-275 inhibited cell survival in dose-dependent manner (Fig. 2A). Next, we performed clonogenic assay to determine the long-term growth inhibitory effect of BML-275. Cells were treated with various concentrations of BML-275 $(0,1,3,5$ and/or $10 \mu \mathrm{M})$ for 1 day and continuously cultured in fresh media for 14 days and colony formation was measured by clonogenic assay. BML-275 significantly inhibited colony formation in dose-dependent manner and at $10 \mu \mathrm{M}$ BML-275 all of the tested pancreatic cancer cells showed susceptibility to the AMPK inhibitor (Fig. 2B). MIA $\mathrm{PaCa}-2$ cells showed increased sensitivity to BML-275 and Panc-1 cells showed relatively less sensitive to BML-275 among four pancreatic cancer cell lines tested (Fig. 2A and B).

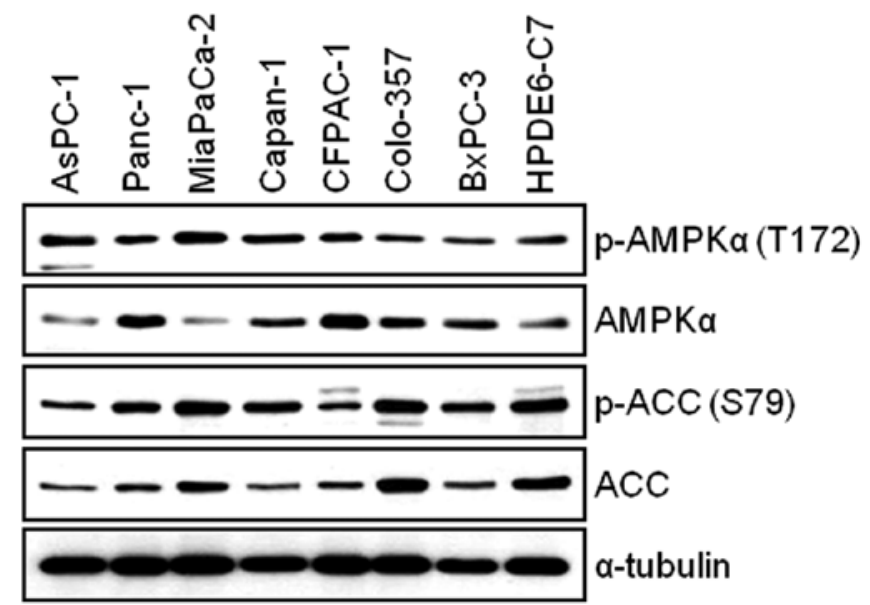

Figure 1. Measurements of AMPK activity in human pancreatic cancer cell lines (AsPC-1, Panc-1, MIA-PaCa2, Capan-1, CFPAC-1, Colo-357 and BxPC-3) and immortal human pancreatic duct epithelial cell line (HPDE6-C7). To avoid bias by conditions of cell growth, rapid growing pancreatic cancer cells were used for WB analysis. The total and phosphorylated forms of AMPK $\alpha$ (T172) and phosphorylated forms of ACC (S79) were determined. The specific phosphorylation site(s) of each kinase is indicated in parentheses. Anti- $\alpha$-tubulin antibody was used for a loading and transfer control.

To investigate mechanism of apoptosis by BML-275 treatment, MIA PaCa- 2 and Panc-1 cells were treated with various

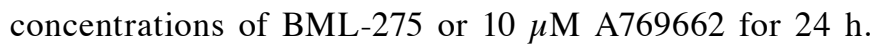
Apoptotic cell death was detected by WB analysis of a molecular biomarker of apoptosis, PARP cleavage. On the contrary to cells treated with A769662, cells treated with BML-275 showed an increase of cleaved PARP in MIA PaCa- 2 cells but not in Panc-1 cells (Fig. 3). However, BML-275 treatment decreased the expression of anti-apoptotic proteins such as Survivin, Bcl2 and XIAP in both cell lines (Fig. 3).

BML-275 induces G2/M arrest and sub-G1. We next investigated if the pharmacological inhibition of AMPK by BML-275 can affect the cell cycle progression in pancreatic cancer cell lines. MIA PaCa-2 and Panc-1 cells were treated with $10 \mu \mathrm{M}$ BML-275 for $24 \mathrm{~h}$ and their cell cycle profiles were assessed by FACS analysis. BML-275 treatment significantly increased the cell population at $\mathrm{G} 2 / \mathrm{M}$-phase (from 15.9 to $58.7 \%$ in MIA $\mathrm{PaCa}-2$ and from 19.4 to $42 \%$ in Panc-1) and significantly decreased the cell population at G0/G1-phase (from 52.8 to $26.4 \%$ in MIA PaCa-2 and from 44.9 to $32.4 \%$ in Panc-1) and S-phase (from 31.3 to $14.9 \%$ in MIA PaCa-2 cells and from 35.7 to $25.6 \%$ in Panc-1) (Fig. 4A). Moreover, we also observed increase of sub-G1 populations. BML-275 increased the sub-G1 population in Panc-1 (from 3 to $7.5 \%$ ) and more significantly in MIA PaCa-2 (from 6.2 to 45.9\%) (Fig. 4A).

DNA damage sensor $\mathrm{CHK} 1 / \mathrm{CHK} 2$ plays a role in G2/M checkpoint via the ataxia-telangiectasia mutated (ATM)/ATM-RAD3-related (ATR) pathway. In order to further elucidate the molecular mechanism leading to BML-275-mediated G2/M arrest, we determined the activation of DNA damage signaling pathway. Treatment of MIA PaCa-2 and Panc-1 cells with BML-275 for $24 \mathrm{~h}$ increased the phosphorylation of ATM at Ser1981 and CHK2 at Thr68 in 
A

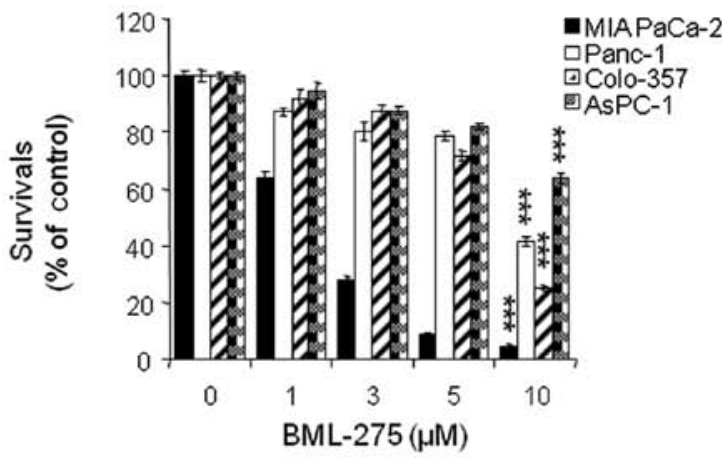

B
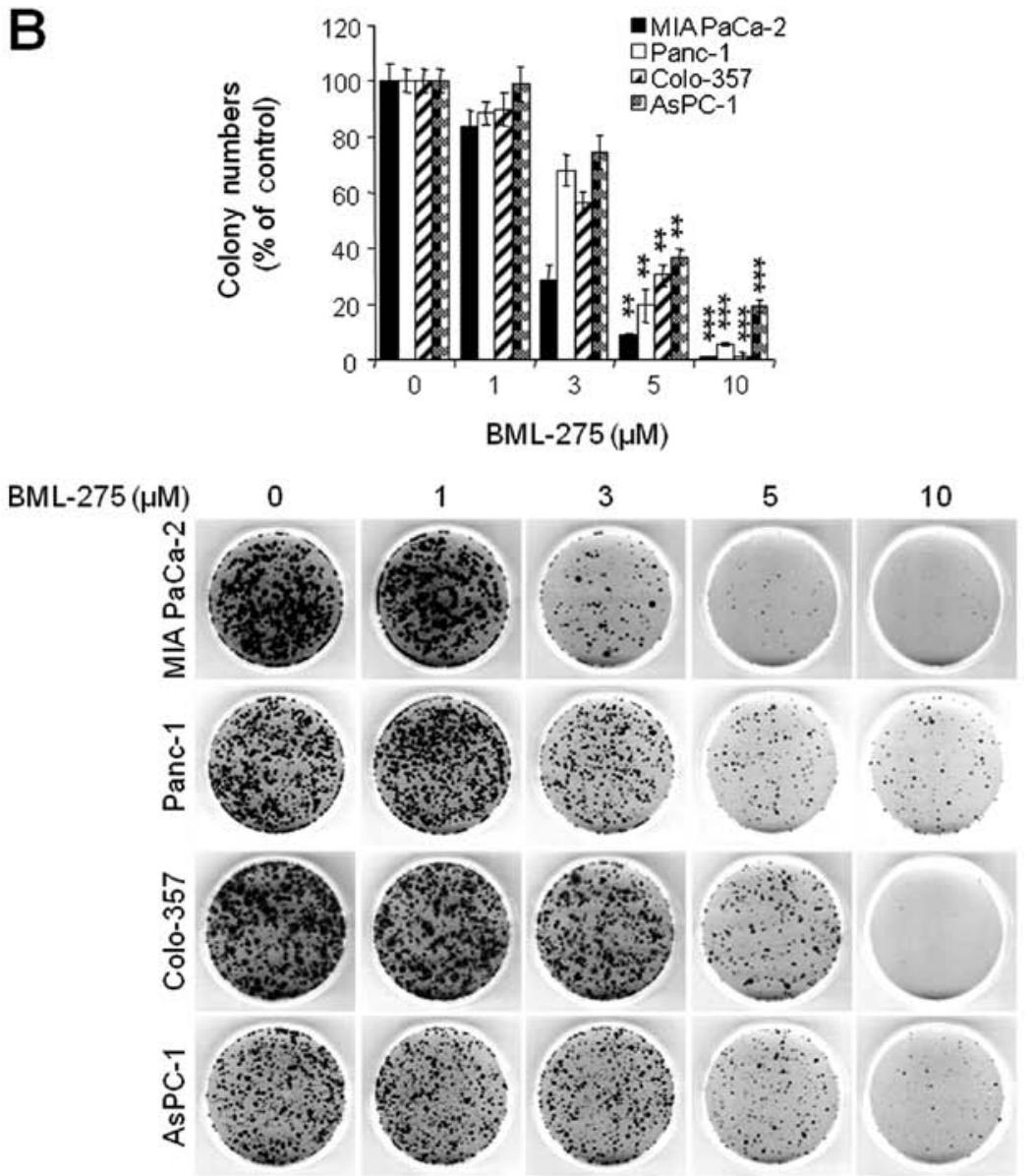

Figure 2. BML-275 inhibits cell viability in human pancreatic cancer cells. (A) An MTT assay of MIA PaCa-2, CFPAC-1, Panc-1 and AsPC-1 cells treated with various concentrations of BML-275 $(0,1,3,5$ and/or $10 \mu \mathrm{M})$ for $48 \mathrm{~h}$ were used to determine cell viability. Error bars represent the standard deviation. ${ }^{* * * *}$ Represents statistically significant difference with p-value $<0.005$ between $10 \mu \mathrm{M}$ BML-275 treated group and control group. (B) A clonogenic assay of MIA PaCa-2, CFPAC-1, Panc-1 and AsPC-1 cells treated with BML-275 $(0,1,3,5$ and/or $10 \mu \mathrm{M})$ for $24 \mathrm{~h}$ was used to determine the long-term response. Colony numbers were counted and calculated as a relative percentage (\%) of the untreated control cells (upper) and representative photograph of clonogenic assay results are shown (lower). Experiments were repeated 3 times and similar results were obtained. Error bars represent the standard deviation. ${ }^{* *}$ Represents statistically significant difference with p-value $<0.01$ between $5 \mu \mathrm{M}$ BML-275 treated group and control group and ${ }^{* * * *}$ represents statistically significant difference with p-value $<0.005$ between $10 \mu \mathrm{M}$ BML-275 treated group and control group.

dose-dependent manner (Fig. 4B). These results coincide with cell cycle arrest in both cell lines. However, the phosphorylation of Histone H2A.X at Ser139, which is the molecular marker of DNA double-strand breaks, more significantly increased in MIA PaCa-2 than in Panc-1 cells (Fig. 4B). Increased levels of CHK2 and H2A.X phosphorylation were more obvious in MIA PaCa-2 cells (Fig. 4B). On the contrary, cells treated by $10 \mu \mathrm{M}$ A769662 for $24 \mathrm{~h}$ did not induce the phosphorylation levels of ATM, CHK2 or Histone H2A.X in either cell line.
BML-275 decreases AMPKa activity in human pancreatic cancer cells. In order to determine the decrease in cell survival and increase in apoptotic cell death closely correlates with the level of inhibition of AMPK activity, cells were pretreated with $10 \mu \mathrm{M}$ A769662 for $6 \mathrm{~h}$ and administered with various concentrations of BML-275 for $24 \mathrm{~h}$. The treatment of $10 \mu \mathrm{M}$ A769662 for $6 \mathrm{~h}$ without BML-275 significantly activated accumulation of phosphorylated levels of AMPK $\alpha$ and ACC in both cell lines (Fig. 5). However, BML-275 treatment reduced 


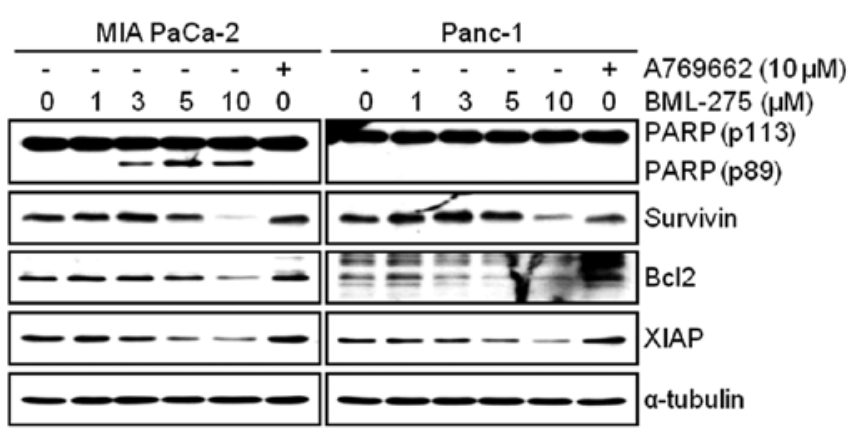

Figure 3. BML-275 induces apoptotic cell death. MIA PaCa-2 and Panc-1 cells were treated with BML-275 $(0,1,3,5$ and/or $10 \mu \mathrm{M})$ or $10 \mu \mathrm{M} \mathrm{A769662} \mathrm{for}$ $24 \mathrm{~h}$. WB analysis for the detection of cleaved PARP(p89) and anti-apoptotic proteins including Survivin, Bcl2 and XIAP was used to measure apoptotic cell death. The level of $\alpha$-tubulin indicates a loading and transfer control.

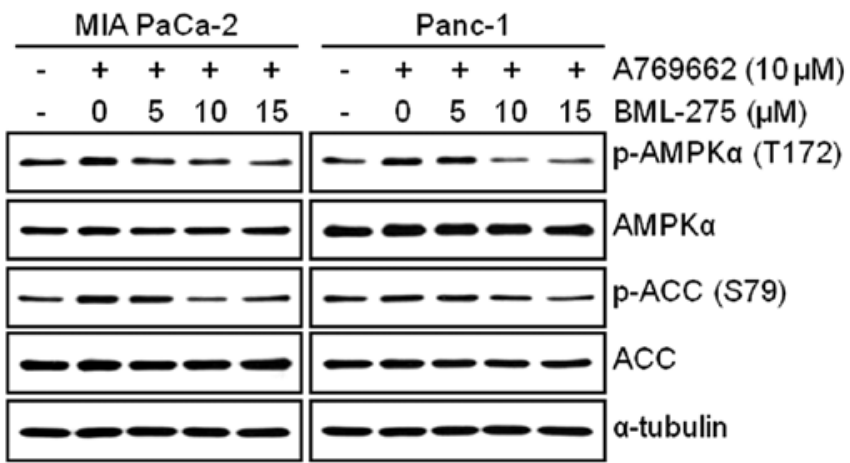

Figure 5. BML-275 inhibits AMPK activity. WB of MIA PaCa-2 and Panc-1 cells pretreated with $10 \mu \mathrm{M}$ A769662 for $6 \mathrm{~h}$ and further treated with BML-275 in different concentrations $(0,5,10$ and/or $15 \mu \mathrm{M})$ for $24 \mathrm{~h}$ were used to determine the total and phosphorylated forms of AMPK $\alpha$ and ACC. Anti- $\alpha$-tubulin antibody was used for a loading and transfer control.

A

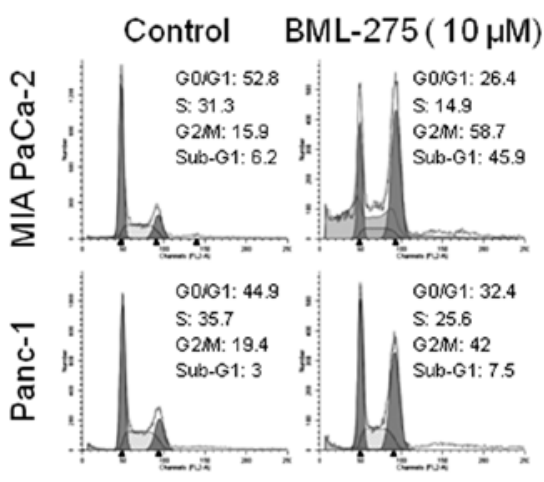

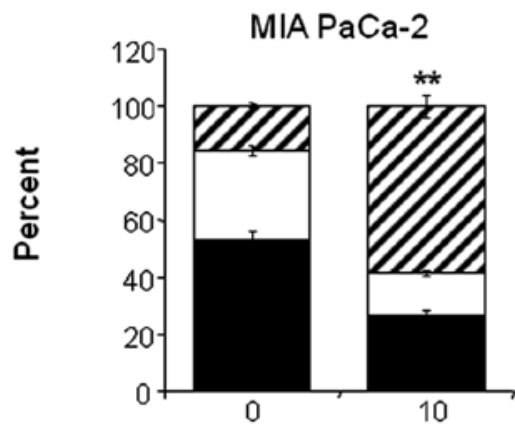

$B M L-275(\mu M)$

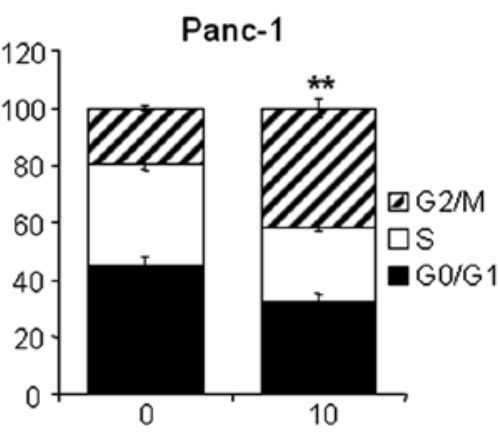

BML-275 $(\mu \mathrm{M})$

B

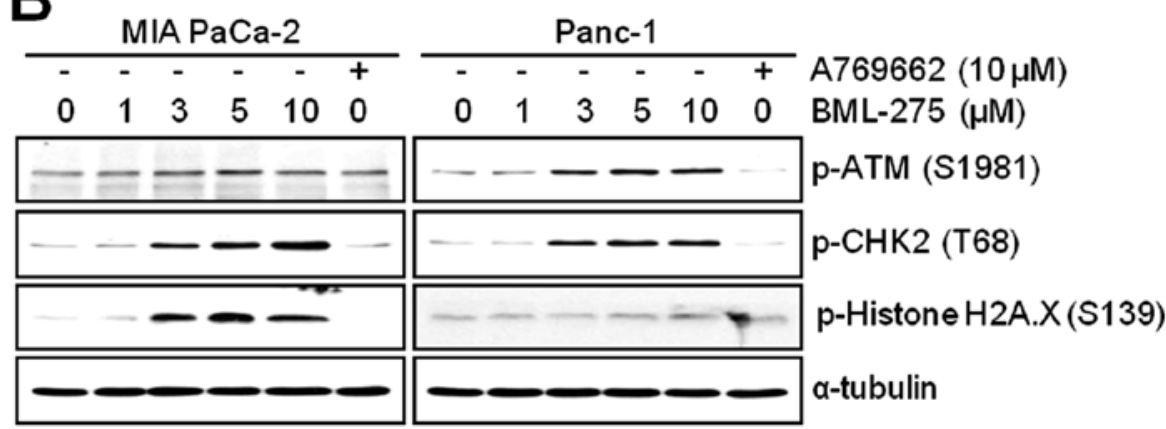

Figure 4. BML-275 induces G2/M arrest and Sub-G1. (A) Analysis by FACS of MIA PaCa-2 and Panc-1 cells treated with $10 \mu \mathrm{M}$ BML-275 for $24 \mathrm{~h}$ was used to determine cell cycle arrest. Experiments were repeated 3 times and similar results were obtained. ${ }^{* *}$ Represents statistically significant difference with p-value $<0.01$ between $10 \mu \mathrm{M}$ BML-275 treated group and control group. (B) A WB of MIA PaCa-2 and Panc-1 cells treated with various concentrations of BML-275 (0, $1,3,5$ and/or $10 \mu \mathrm{M}$ ) or $10 \mu \mathrm{M}$ A769662 for $24 \mathrm{~h}$ was used to determine the phosphorylated forms of ATM (S1981), CHK2 (T68) and Histone H2A.X (S139). The specific phosphorylation site(s) of each kinase is indicated in parentheses. Anti- $\alpha$-tubulin antibody was used for a loading and transfer control. 
A

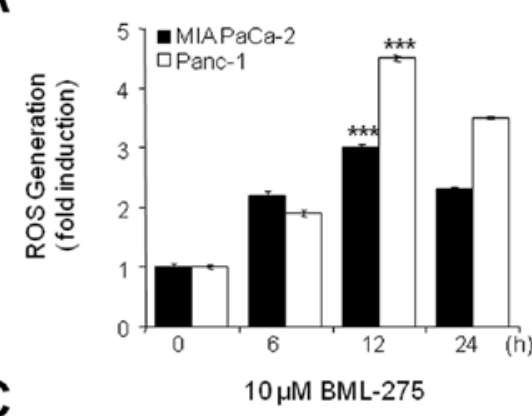

C

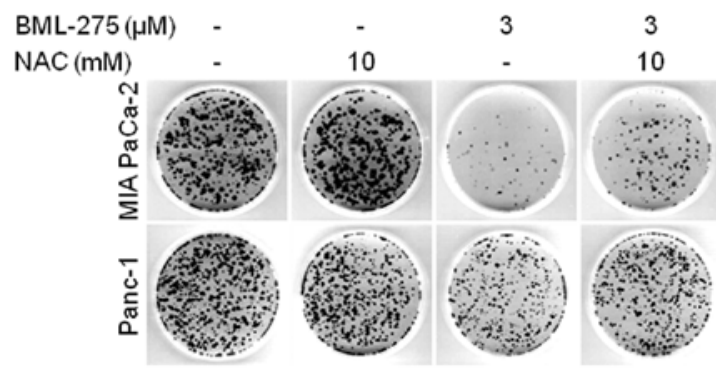

B

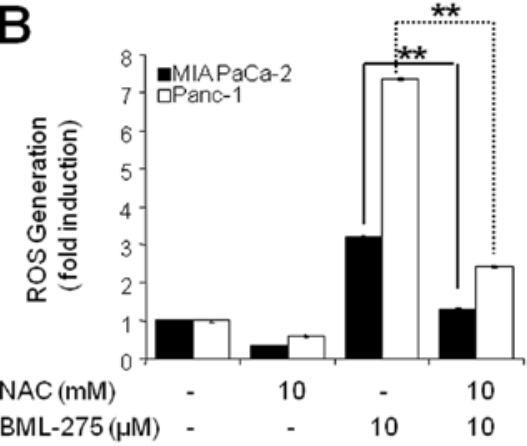

\section{D}

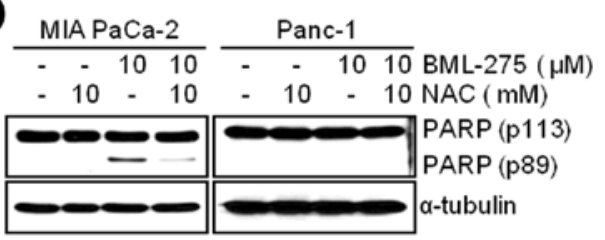

E
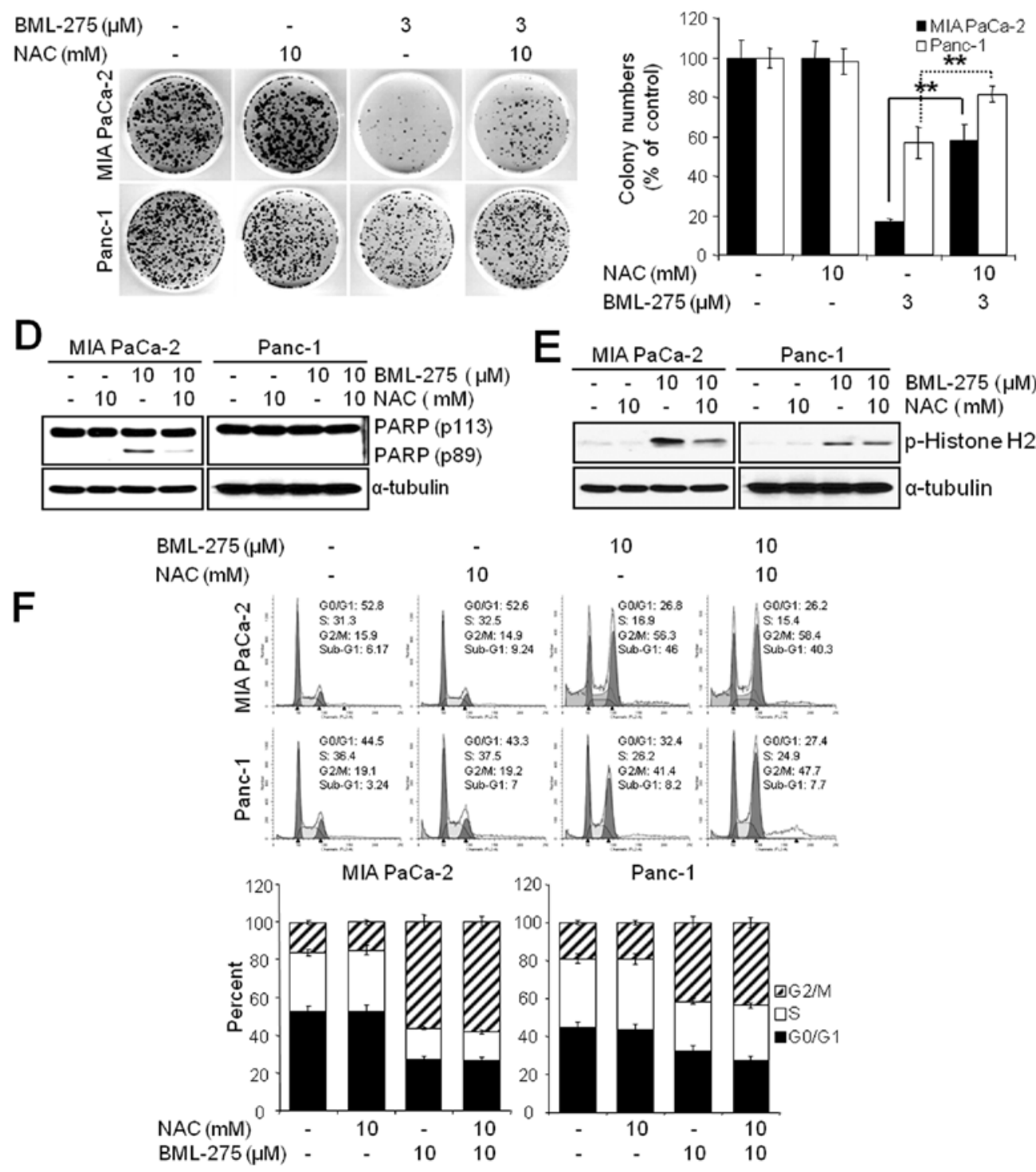

Figure 6. ROS generation is critically required for apoptotic cell death by BML-275. (A) The result of ROS generation analysis of MIA PaCa-2 and Panc-1 cells treated with $10 \mu \mathrm{M}$ BML-275 in different time intervals $(0,6,12$ and/or $24 \mathrm{~h})$ and treated with DCFDA for a further $0.5 \mathrm{~h}$ to measure ROS generation. Experiments were repeated 3 times and similar results were obtained. Error bars represent the standard deviation. ${ }^{* * *}$ Represents statistically significant difference with p-value $<0.005$ between $10 \mu \mathrm{M}$ BML-275 $12 \mathrm{~h}$ treated group and control group. (B) The result of ROS generation analysis of MIA PaCa- 2 and Panc- 1 cells pretreated with $10 \mathrm{mM}$ NAC for $1 \mathrm{~h}$ and further treated with $10 \mu \mathrm{M}$ BML-275 for $12 \mathrm{~h}$ and then treated with DCFDA for further $0.5 \mathrm{~h}$ to measure ROS generation. Experiments were repeated 3 times and similar results were obtained. Error bars represent the standard deviation. ${ }^{* *}$ Represents statistically significant difference with p-value $<0.01$ between $10 \mu \mathrm{M}$ BML-275 treated group and $10 \mathrm{mM}$ NAC plus $10 \mu \mathrm{M}$ BML-275 group. (C) A clonogenic assay of MIA PaCa-2 and Panc-1 cells pretreated with $10 \mathrm{mM} \mathrm{NAC}$ for $1 \mathrm{~h}$ and further treated with $3 \mu \mathrm{M}$ BML-275 for $24 \mathrm{~h}$ was used to determine the long-term response. Colony numbers were counted and calculated as a relative percentage (\%) of the untreated control cells (left) and representative photograph of colony formation assay results are shown (right). Experiments were repeated 3 times and similar results were obtained. Error bars represent the standard deviation. ${ }^{* *}$ Represents statistically significant difference with p-value $<0.01$ between $10 \mathrm{mM}$ NAC plus $3 \mu \mathrm{M}$ BML-275 and $3 \mu \mathrm{M}$ BML-275 group. (D) A WB analysis of MIA PaCa-2 and Panc-1 cells pretreated with 10 mM NAC for $1 \mathrm{~h}$ and further treated with $10 \mu \mathrm{M}$ BML-275 for $24 \mathrm{~h}$ was used to determine apoptotic cell death by anti-cleaved PARP antibody. (E) A WB analysis of MIA PaCa-2 and Panc-1 cells pretreated with $10 \mathrm{mM} \mathrm{NAC}$ for $1 \mathrm{~h}$ and further treated with $10 \mu \mathrm{M}$ BML-275 for $24 \mathrm{~h}$ was used to determine the phosphorylated forms of Histone H2A.X (S139). The specific phosphorylation site(s) of each kinase is indicated in parentheses. (F) Analysis by FACS of MIA PaCa-2 and Panc-1 cells pretreated with $10 \mathrm{mM}$ NAC for $1 \mathrm{~h}$ and treated with $10 \mu \mathrm{M}$ BML-275 for $24 \mathrm{~h}$ was used to determine cell cycle arrest. Experiments were repeated 3 times and similar results were obtained. Error bars represent the standard deviation. 

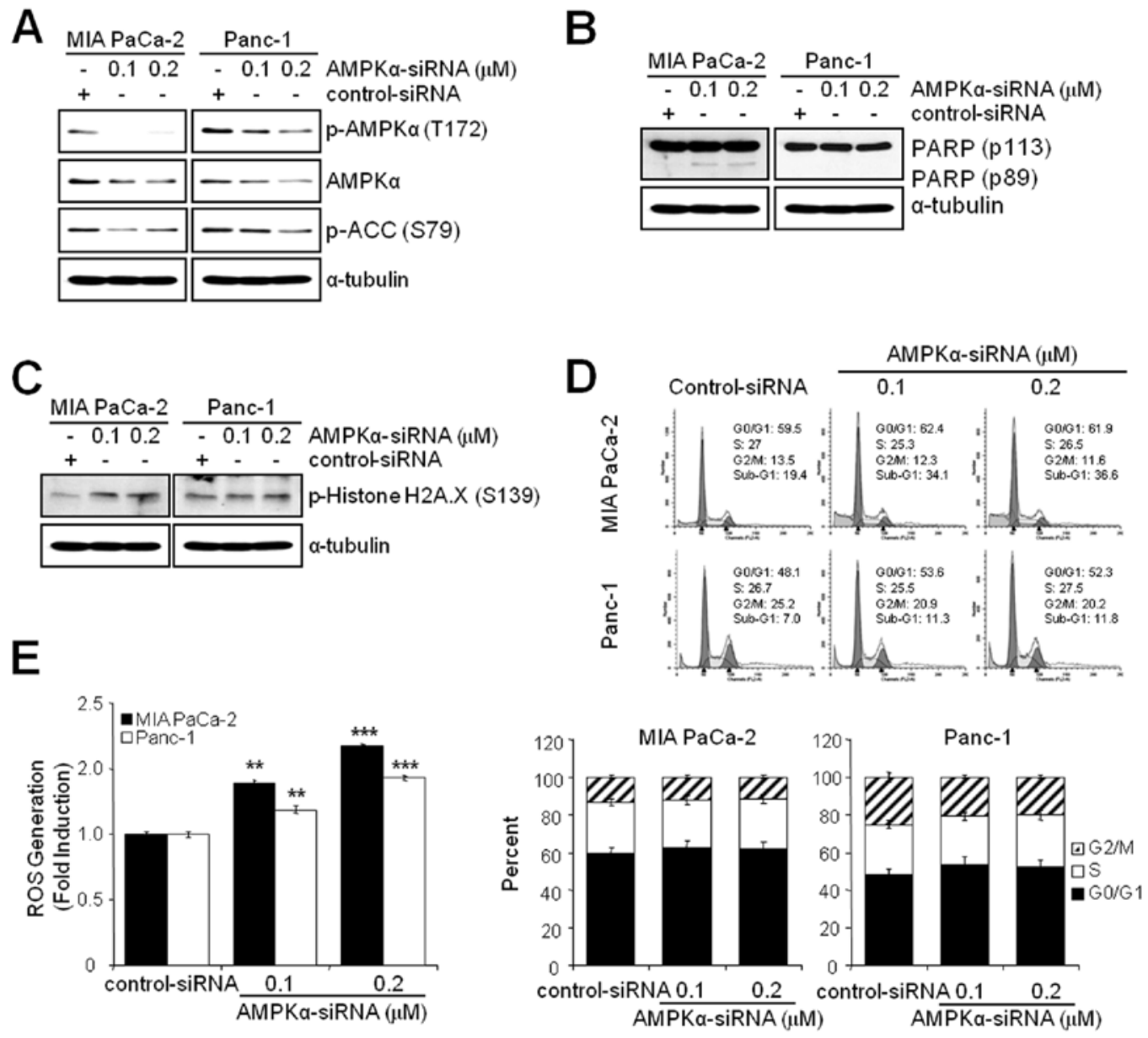

Figure 7. Knockdown of AMPK $\alpha$ induces ROS generation and apoptosis but not G2/M arrest. MIA PaCa- 2 and Panc- 1 cells were transfected with Control- and AMPK $\alpha$-siRNA for $48 \mathrm{~h}$ as described in Materials and methods. (A) WB analysis was used to determine the total and phosphorylated forms of AMPK $\alpha$ and phosphorylated forms of ACC. (B) WB analysis for the detection of PARP cleavage was used to measure apoptotic cell death. (C) WB analysis was used to determine the phosphorylated forms of Histone H2A.X (S139). The specific phosphorylation site(s) of each kinase is indicated in parentheses. (D) Analysis by FACS was used to determine cell cycle arrest. Experiments were repeated 3 times and similar results were obtained. Error bars represent the standard deviation. (E) ROS generation analysis was used to measure ROS generation. Experiments were repeated 3 times and similar results were obtained. Error bars represent the standard deviation. ${ }^{* * *}$ Represents statistically significant difference of ROS generation with p-value $<0.01$ between AMPK $\alpha$-siRNA $(0.1 \mu \mathrm{M})$ and control-siRNA group. ${ }^{* * *}$ Represents statistically significant difference of ROS generation with p-value $<0.005$ between AMPK $\alpha$-siRNA $(0.2 \mu \mathrm{M})$ and control-siRNA group.

the phosphorylation of AMPK $\alpha$ and ACC exerted by A769662 in a dose-dependent manner (Fig. 5), suggesting that antitumor effect(s) by BML-275 closely correlates with the level of inhibition of AMPK activity in human pancreatic cancer cell lines.

The generation of ROS by BML-275 is critically required for the induction of cell death but not G2/M arrest. Since oxidative stress is a potent inducer of apoptosis, we next investigated if BML-275 could cause a generation of ROS in pancreatic cancer cell lines. We determined ROS generation by measuring the fluorescence of DCF which is formed by the oxidation of DCFDA by peroxides. Our results demonstrated early ROS generation by BML-275 in both cell lines (Fig. 6A). BML-275induced ROS generation was significantly diminished by incubation with the antioxidant agent, NAC (Fig. 6B). NAC also rescued BML-275-mediated inhibition of cell survival by MTT assay (data not shown) and clonogenic assay (Fig. 6C). It also relieved the cleavage of PARP by BML-275 treatment in MIA PaCa-2 cells (Fig. 6D). BML-275-mediated phosphorylation of H2A.X at Ser139 also inhibited by NAC pretreatment
(Fig. 6E). However, NAC administration did not alleviate G2/M arrest induced by BML-275 treatment (Fig. 6F), suggesting that BML-275-mediated G2/M arrest is ROS-independent at least in pancreatic cancer cell lines used for this study.

Knockdown of AMPKa induces ROS generation and apoptosis but not G2/M arrest. Since the inhibition of AMPK by BML-275 induced DNA damage, G2/M arrest and apoptosis in human pancreatic cancer cells, MIA PaCa-2 and Panc-1 cells were transfected with control-siRNA or AMPK $\alpha$-siRNA to compare the effect(s) of BML-275 and knockdown of AMPK $\alpha$. Knockdown of AMPK $\alpha$ with concentration of 0.1 or $0.2 \mu \mathrm{M}$ AMPK $\alpha$-siRNA suppressed the level of total and phosphorylated form of AMPK $\alpha$ and phosphorylated form of ACC in MIA PaCa-2 and Panc-1 cells (Fig. 7A). In addition, knockdown of AMPK $\alpha$ also induced apoptotic cell death as evidenced by induction of PARP cleavage in MIA-PaCa-2 cells (Fig. 7B) and accumulation of sub-G1 cells in FACS analysis in MIA PaCa-2 cells (from $19.4 \%$ by control to $34.1 \%$ by $0.1 \mu \mathrm{M}$ AMPK $\alpha$ siRNA) but to a lesser extent in Panc-1 cells (from $7.0 \%$ by control to 


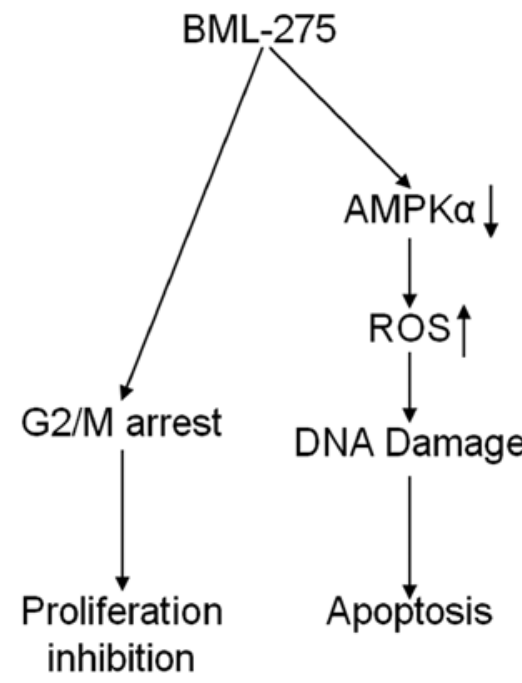

Figure 8 . The proposed model for the mechanism by action of BML-275 in human pancreatic cancer cells. BML-275 induced DNA damage and apoptosis that is crucially regulated by ROS generation. Moreover, BML-275 caused cell cycle arrest at $\mathrm{G} 2 / \mathrm{M}$-phase.

$11.3 \%$ by $0.1 \mu \mathrm{M}$ AMPK $\alpha$-siRNA (Fig. 7D). Knockdown of $\mathrm{AMPK} \alpha$ in MIA PaCa-2 cells also induced phosphorylation of H2A.X at Ser139 indicating DNA damage (Fig. 7C). The Panc-1 cells show resistance to phosphorylation of H2A.X similarly to BML-275 treatment (Fig. 4B). However, knockdown of AMPK $\alpha$ activity fails to display a cell cycle arrest in G2/M-phase in MIA PaCa-2 and Panc-1 cells (Fig. 7D). Finally, AMPK $\alpha$ knockdown induced ROS generation with increasing concentration of AMPK $\alpha$-siRNA (Fig. 7E). Taken together, in pancreatic cancer cell lines, targeting of AMPK $\alpha$ is able to induce DNA damage, ROS generation and apoptotic cell death but not G2/M arrest.

\section{Discussion}

In this study, we investigated the molecular mechanism of antitumor effect(s) of BML-275, an AMPK inhibitor, in human pancreatic adenocarcinoma. We found that: i) the levels of total and phosphorylated form of AMPK $\alpha$ and ACC vary in several different human pancreatic cancer cell lines; ii) BML-275 inhibits cell proliferation in MIA PaCa-2, Panc-1, Colo-357 and AsPC-1 cells; iii) BML-275 induces DNA damage, apoptosis and $\mathrm{G} 2 / \mathrm{M}$ arrest; iv) the ROS generation by BML-275 is critically required for the DNA damage and apoptosis but not G2/M arrest and v) knockdown of AMPK $\alpha$ induces ROS generation, DNA damage and apoptosis but not G2/M arrest. This is the first report showing that BML-275 induces DNA damage, G2/M arrest and apoptosis in pancreatic cancer cell lines.

AMPK is a survival factor for cancer cells. It is involved in the augmentation of energy production through the activation of glucose uptake, glycolysis and fatty acid oxidation in response to ATP-depleting stresses (38). Solid tumors outgrowing the existing vasculature are continuously exposed to a microenvironment in which the supply of both oxygen and nutrition is limited. Previous studies showed that AMPK is critical for cancer cell adaptation in response to hypoxia or glucose deprivation (39-42). The protective role of AMPK is not restricted to nutrient stress, as this enzyme seems to play an important role in protecting tumor cells from apoptosis induced by chemotherapeutic agents such as doxorubicin, cisplatin and TRAIL (24-26). In addition, pharmacological inhibition of AMPK by BML-275 induced apoptotic cell death in myeloma, glioma, prostate cancer and breast carcinoma cells (20-23). Moreover, transfection with dominant-negative AMPK or AMPK $\alpha$-siRNA was also sufficient to reduce cell proliferation of BHK, HeLa and PC12 pheochromocytoma cells or CWR22Rv1 and LNcaP prostate cancer cells $(21,43)$. Comparing with effective apoptosis inducing dose of BML-275 treated in other cancer cell lines reported previously (20-23), most of the pancreatic cancer cell lines responded to BML-275 with different levels of responsiveness. Pancreatic cancer cell lines with relatively high level of phosphorylated AMPK showed more susceptibility to BML-275 treatment (MIA PaCa-2 and Colo-357), and those with low phosphorylated AMPK showed relatively decreased sensitivity (Panc-1 and AsPc-1).

Cancer cells usually exhibit increased levels on intracellular ROS, which in turn can initiate various cycles leading to further metabolic malfunction and ROS generation $(44,45)$. ROS cause oxidative damage to DNA, proteins, lipids and other cellular components and therefore also significant cellular stress (45). A proposed therapeutic strategy against cancer is to treat cancer cells with pharmacological agents that have pro-oxidant properties which increase the intracellular ROS generation to a toxic threshold that triggers cell death in the cancer cells without harming normal cells (44). Vuvicevic et al showed that BML-275 induces ROS generation in glioma cell line, but AMPK $\alpha$-siRNA treatment fails to induce ROS generation and apoptosis (22). In this study, an increased generation of ROS upon either BML-275 or AMPK $\alpha$-siRNA treatment was observed and the intracellular accumulation of ROS seems to be one of critical factors in BML-275-induced apoptosis. To verify this speculation, NAC, scavenger of oxygen-free radicals, was challenged with BML-275. NAC relieved BML-275 or AMPK-siRNA mediated ROS production and improved cell viability based on the clonogenic assay, which suggested that both chemical and genetic inhibitor regulate cell viability via repressing AMPK activity.

The G2/M checkpoint plays an important role in cellular response to genotoxic stimuli. The G2/M checkpoint prevents cells from entering mitosis when DNA is damaged, providing an opportunity for repair and stopping the proliferation of damaged cells which help to maintain genomic stability (46). CHK1 and CHK2 kinases are activated at G2-phase checkpoint by DNA damage or unreplicated chromosomal DNA (47), and inactivate $\mathrm{Cdc} 25 \mathrm{C}$ through its phosphorylation $(48,49)$. Cdc $25 \mathrm{C}$ was the protein phosphatase responsible for dephosphorylating and activating $\mathrm{Cdc} 2$, a crucial step in regulating the entry of all eukaryotic cells into the M-phase of the cell cycle. In this study, BML-275 induces cell cycle arrest at G2/M-phase possibly through the phosphorylation and activation of $\mathrm{CHK} 2$ kinase. The pretreatment of NAC restores the generation of ROS by BML-275 treatment in MIA PaCa- 2 cell line, however, the cell cycle arrest at $\mathrm{G} 2 / \mathrm{M}$ phase cannot be relieved, suggesting unknown effects of BML-275 or non-target effects may play a role in G2/M arrest. Previously AMPK $\alpha$-siRNA treatment was reported to induce $\mathrm{G} 2 / \mathrm{M}$ arrest in the absence of ROS generation and with no apparent cell death in U251 glioma cells (22). However, in pancreatic cancer cell line, the AMPK $\alpha$-siRNA 
treatment induces generation of ROS and apoptotic cell death but no apparent G2/M arrest. Thus, our finding suggests that pancreatic cancer cells may be able to override the cell cycle arrest $(\mathrm{G} 2 / \mathrm{M})$ in response to AMPK knockdown by siRNA. On the other hand, the mechanism of DNA damage and cell death induced by BML-275 seems to be via inhibition of AMPK activity followed by stimulation of ROS production. Panc-1 is known as relatively more resistant to various antitumor agents among several pancreatic cancer cell lines (50-52). Our study also show panc-1 as more resistant to apoptotic response (cell death and PARP cleavage) upon the treatment of BML-275 and AMPK $\alpha$-siRNA. Although we could not demonstrate the mechanism of resistance of Panc-1 to BML-275 treatment, this may be due to its increased multidrug resistance (MDR) gene products and/or constitutively activated cell surviving signaling pathways that confer intrinsic drug resistance (50-54).

In conclusion, our findings implicate that BML-275 induces DNA damage and apoptosis through AMPK-dependent mechanism and induces G2/M arrest through AMPK-independent mechanism (Fig. 8). Although the molecular mechanism of antitumor effect(s) by BML-275 requires further investigation, this compound seems to be a novel potential therapeutic agent to treat human pancreatic cancer.

\section{Acknowledgements}

IB was supported by National Institutes of Health (1R03CA152530), the National Research Foundation of Korea [R31-10069; World Class University (WCU) program] and the Georgetown University Lombardi Comprehensive Cancer Center (P30-CA051008).

\section{References}

1. Jemal A, Siegel R, Xu J and Ward E: Cancer statistics, 2010. CA Cancer J Clin 60: 277-300, 2010.

2. Keighley MR: Gastrointestinal cancers in Europe. Aliment Pharmacol Ther 18: 7-30, 2003.

3. Hardie DG: AMP-activated/SNF1 protein kinases: conserved guardians of cellular energy. Nat Rev Mol Cell Biol 8: 774-785, 2007.

4. Hardie DG and Carling D: The AMP-activated protein kinase - fuel gauge of the mammalian cells? Eur J Biochem 246 : 259-273, 1997

5. Hardie DG, Scott JW, Pan DA and Hudson ER: Management of cellular energy by the AMP-activated protein kinase system. FEBS Lett 546: 113-120, 2003.

6. Carling D: The AMP-activated protein kinase cascade - a unifying system for energy control. Trends Biochem Sci 29: $18-24,2004$

7. Hardie DG: The AMP-activated protein kinase pathway - new players upstream and downstream. J Cell Sci 117: 5479-5487, 2004.

8. Kahn BB, Alquier T, Carling D and Hardie DG: AMP-activated protein kinase: ancient energy gauge provides clues to modern understanding of metabolism. Cell Metab 1: 15-25, 2005.

9. Hawley SA, Pan DA, Mustard KJ, Ross L, Bain J, Edelman AM, Frenguelli BG and Hardie DG: Calmodulin-dependent protein kinase kinase-beta is an alternative upstream kinase for AMP-activated protein kinase. Cell Metab 2: 9-19, 2005.

10. Hurley RL, Anderson KA, Franzone JM, Kemp BE, Means AR and Witters LA: The $\mathrm{Ca}^{2+} /$ calmodulin-dependent protein kinase kinases are AMP-activated protein kinase kinases. J Biol Chem 280: 29060-29066, 2005.

11. Woods A, Dickerson K, Heath R, Hong SP, Momcilovic M, Johnstone SR, Carlson M and Carling D: $\mathrm{Ca}^{2+} /$ calmodulin-dependent protein kinase kinase-beta acts upstream of AMP-activated protein kinase in mammalian cells. Cell Metab 2: 21-33, 2005.
12. Campàs C, Lopez JM, Santidrián AF, Barragán M, Bellosillo B, Colomer D and Gil J: Acadesine activates AMPK and induces apoptosis in B-cell chronic lymphocytic leukemia cells but not in T lymphocytes. Blood 101: 3674-3680, 2003.

13. Kefas BA, Cai Y, Kerchhofs K, Ling Z, Martens G, Heimberg H, Pipeleers D and Van de Casteele M: Metformin-induced stimulation of AMP-activated protein kinase in beta-cells impairs their glucose responsiveness and can lead to apoptosis. Biochem Pharmacol 68: 409-416, 2004.

14. Saitoh M, Nagai K, Nakagawa K, Yamamura T, Yamamoto $S$ and Nishizaki T: Adenosine induces apoptosis in the human gastric cancer cells via an intrinsic pathway relawant to activation of AMP-activated protein kinase. Biochem Pharmacol 67: 2005-2011, 2004

15. Rattan R, Giri S, Singh AK and Singh I: 5-aminoimidazole4-carboxamide-1-beta-D-ribofuranoside inhibits cancer cell proliferation in vitro and in vivo via AMP-activated protein kinase. J Biol Chem 280: 39582-39593, 2005.

16. Zhou W, Han WF, Landree LE, Thupari JN, Pinn ML, Bililign T, Kim EK, Vadlamudi A, Medghlchi SM, El Meskini R, Ronnett GV, Townsend CA and Kuhajda FP: Fatty acid synthase inhibition activates AMP-activated protein kinase in SKOV3 human ovarian cancer cells. Cancer Res 67: 2964-6971, 2007.

17. Isakovic A, Harhaji L, Stevanovic D, Markovic Z, SumaracDumanovic M, Starcevic V, Micic D and Trajkovic V: Dual antiglioma action of metformin: cell cycle arrest and mitochondria-dependent apoptosis. Cell Mol Life Sci 64: 1290-1302, 2007.

18. Okoshi R, Ozaki T, Yamamoto H, Ando K, Koida N, Ono S, Koda T, Kamijo T, Nakagamara A and Kizaki H: Activation of AMP-activated protein kinase induces p53-dependent apoptotic cell death in response to energetic stress. J Biol Chem 283: 3979-3987, 2008.

19. Senqupta TK, Leclerc GM, Hsieh-Kinser TT, leclerc GJ, Singh I and Barredo JC: Cytotoxic effect of 5-aminoimidazole-4-carboxamide-1-beta-D-ribofuranoside (AICAR) on childhood acute lymphoblastic leukemia (ALL) cells: implication for targeted therapy. Mol Cancer 6: 46, 2007.

20. Baumann P, Mandl-Weber S, Emmerich B, Straka C and Schmidmaier R: Inhibition of adenosine monophosphateactivated protein kinase induces apoptosis in multiple myeloma cells. Anticancer Drugs 18: 405-410, 2007.

21. Park HU, Suy S, Danner M, Dailey V, Zhang Y,Li H, Hyduke DR, Collins BT, Gagnon G, Kallakury B, Kumar D, Brown ML, Fornace A, Dritschilo A and Collins SP: AMP-activated protein kinase promotes human prostate cancer cell growth and survival. Mol Cancer Ther 8: 733-741, 2009.

22. Vuvicevic L, Misirkic M, Janjetovic K, Harhaji-Trajkovic L, Prica M, Stevanovic D, Isenovic E, Sudar E, SumaracDumanovic M, Micic D and Trajkovic V: AMP-activated protein kinase-dependent and-independent mechanisms underlying in vitro antiglioma action of compound $\mathrm{C}$. Biochem Pharmacol 77: 1684-1693, 2009.

23. Jin J, Mullen TD, Hou Q, Bielawski J, Bielawska A, Zhang X, Obeid LM, Hannun YA and Hsu YT: AMPK inhibitor compound $\mathrm{C}$ stiumulates ceramine production and promotes BAx redistribution and apoptosis in MCF-7 breast carcinoma cells. J Lipid Res 50: 2389-2397, 2009.

24. Jang JH, Lee TJ, Yang ES, Min do S, Kim YH, Kim SH, Choi YH, Park JW, Choi KS and Kwon TK: Compound C sensitizes caki renal cancer cells to TRAIL-induced apoptosis through reactive oxygen species-mediated down-regulation of c-FLIPL and Mcl-1. Exp Cell Res 316: 2194-2203, 2010.

25. Zhu Q, Shen B, Zhang B, Zhang W, Chin SH, Jin J and Liao DF: Inhibition of AMP-activated protein kinase pathway sensitizes human leukemia K562 cells to nontoxic concentration of doxorubicin. Mol Cell Biochem 340: 275-281, 2010.

26. Kim HS, Hwang JT, Yun H, Chi SG, Lee SJ, Kang I, Yoon KS, Choe WJ, Kim SS and Ha J: Inhibition of AMP-activated protein kinase sensitizes cancer cells to cisplatin-induced apoptosis via hyper-induction of p53. J Biol Chem 283: 3731-3742, 2008.

27. Collins K, Jacks T and Pavletich NP: The cell cycle and cancer. Proc Natl Acad Sci USA 94: 2776-2778, 1997.

28. Buolamwini JK: Cell cycle molecular targets in novel anticancer drug discovery. Curr Pharm Des 6: 379-392, 2000.

29. Hajduch M, Havlieek L, Vesely J, Novotny R, Mihal V and Strnad M: Synthetic cyclin dependent kinase inhibitors. New generation of potent anti-cancer drugs. Adv Exp Med Biol 457: 341-353, 1999.

30. Pines J: Four-dimensional control of the cell cycle. Nat Cell Biol 1: E73-E79, 1999. 
31. Taylor WR and Stark GR: Regulation of the G2/M transition by p53. Oncogene 20: 1803-1815, 2001.

32. Ueda S, Nakamura H, Masutani H, Sasada T, Takabayashi A, Yamaoka Y and Yodoi J: Baicalin induces apoptosis via mitochondrial pathway as prooxidant. Mol Immunol 38: 781-791, 2002.

33. Beckman KB and Ames BN: Oxidative decay of DNA. J Biol Chem 272: 19633-19636, 1997

34. Cadet J, Delatour T, Douki T, Gasparutto D, Pouget JP, Ravanat JL and Sauvaigo S: Hydroxyl radicals and DNA base damage. Mutal Res 424: 9-21, 1999.

35. Vilenchik MM and Knudson AG: Endogenous DNA doublestrand breaks:production, fidelity of repair and induction of cancer. Proc Natl Acad Sci USA 100: 12871-12876, 2003

36. Furukawa T, Duquid WP, Rosenberg L, Viallet J, Galloway DA and Tsao MS: Long-term culture and immortalization of epithelial cells from normal adult human pancreatic ducts transfected by the E6E7 gene of human papilloma virus 16. Am J Pathol 148: 1763-1770, 1996

37. Duong HQ, Kim HJ, Kang HJ, Seong YS and Bae I: ZSTK474, a PI3K inhibitor, suppresses proliferation and sensitizes human pancreatic adenocarcinoma cells to gemcitabine. Oncol Rep 27: 182-188, 2012.

38. Hardie DG, Carling D and Carlson M: The AMP-activated/ SNF1 protein kinase subfamily: metabolic sensors of the eukaryotic cells? Annu Rev Biochem 67: 821-855, 1998.

39. Lee M, Hwang JT, Lee HJ, Jung SN, Kang I, Chi SG, Kim SS and $\mathrm{Ha} \mathrm{J}$ : AMP-activated protein kinase activity is critical for hypoxia-inducible factor- 1 transcriptional activity and its target gene expression under hypoxic conditions in DU145 cells J Biol Chem 278: 39653-39661, 2003.

40. Yun H, Lee M, Kim SS and Ha J: Glucose deprivation increases mRNA stability of vascular endothelial growth factor through activation of AMP-activated protein kinase in DU145 prostate carcinoma. J Biol Chem 280: 9963-9972, 2005.

41. Kato K, Ogura T, Kishimoto A, Minegishi Y, Nakajima N, Miyazaki M and Esumi H: Critical roles of AMP-activated protein kinase in constitutive tolerance of cancer cells to nutrient deprivation and tumor formation. Oncogene 21: 6082-6090, 2002.

42. Esumi H, Izuishi K, Kato K, Hashimoto K, Kurashima Y, Kishimoto A, Ogura T and Ozawa T: Hypoxia and nitric oxide treatment confer tolerance to glucose starvation in a 5'-AMP-activated protein kinase dependent manner. J Biol Chem 277: 32791-32798, 2002.
43. Shaw MM, Gurr WK, McCrimmon RJ, Schorderer DF and Sherwin RS: 5'AMP-activated protein kinase alpha deficiency enhances stress-induced apoptosis in BHK and PC12 cells. J Cell Mol Med 11: 286-298, 2007.

44. Trachootham D, Alexandre J and Huang P: Targeting cancer cells by ROS-mediated mechanisms: a radical therapeutic approach? Nat Rev Drug Discov 8: 579-591, 2009.

45. Luo J, Solimini NL and Elledqe SJ: Principles of cancer therapy: oncogene and non-oncogene addiction. Cell 136: 823-837, 2009.

46. Lobrich $M$ and Jeggo PA: The impact of a negligent G2/M checkpoint on genomic instability and cancer induction. Nat Rev Cancer 7: 861-869, 2007.

47. Smits VA and Medema RH: Checking out the $\mathrm{G}(2) / \mathrm{M}$ transition. Biochim Biophys Acta 1519: 1-12, 2001.

48. Gotoh T, Ohsumi K, Matsui T, Takisawa $\mathrm{H}$ and Kishimoto $\mathrm{T}$ : Inactivation of the checkpoint kinase Cds1 is dependent on cyclin B-Cdc2 kinase activation at the meiotic G2/M-phase transition in xenopus oocytes. J Cell Sci 114: 3397-3406, 2001.

49. Singh SV, Herman-Antosiewicz A, Singh AV, Lew KL, Srivastava SK, Kamath R, Brown KD, Zhang L and Baskaran R: Sulforaphane-induced G2/M phase cell cycle arrest involves checkpoint kinase 2-mediated phosphorylation of cell division cycle 25C. J Biol Chem 279: 25813-25822, 2004.

50. Huanwen W, Zhiyong L, Xiaohua S, Xinyu R, Kai W and Tonghua L: Intrinsic chemoresistance to gemcitabine is associated with constitutive and laminin-induced phosphorylation of FAK in pancreatic cancer cell lines. Mol Cancer 8: 125, 2009.

51. Kreutzer JN, Ruzzene M and Guerra B: Enhancing chemosensitivity to gemcitabine via RNA interfence targeting the catalytic subunits of protein kinase CK2 in human pancreatic cancer cells. BMC Cancer 10: 40, 2010.

52. Danilov AV, Neupane D, Nagaraja AS, Feofanova EV, Humphries LA, DiRenzo J and Korc M: DeltaNp63alphamediated induction of epidermal growth factor receptor promotes pancreatic cancer cell growth and chemoresistance. PloS One 6: e26815, 2011.

53. Hagmann W, Jesnowski R and Löhr JM: Interdependence of gemcitabine treatment, transporter expression, and resistance in human pancreatic carcinoma cells. Neoplasia 12: 740-747, 2010.

54. Yao Z, Okabayashi Y, Yutsudo Y, Kitamura T, Ogawa W and Kasuga M: Role of Akt in growth and survival of Panc-1 pancreatic cancer cells. Pancreas 24: 42-46, 2002. 